\title{
Die "unsichtbare Hand" in der Sprache. Eine kritische Betrachtung von Kellers Sprachwandeltheorie
}

\author{
Francina Ladstätter (Zürich)
}

\begin{abstract}
How is language change effected and how does it proceed? In this article the central concepts of Rudi Keller's much-quoted work "Sprachwandel: von der unsichtbaren Hand in der Sprache" ("On Language Change. The Invisible Hand in Language") are introduced and the main criticism presented. Following this first part, Keller's theory of language change is related to the concrete example of linguistic changes in job advertisements. The main interest here is in the enhancement of job titles ("Gebrauch von aufwertenden Berufs- und Personenbezeichnungen"). 26,000 job advertisements from 1950 to 1999 are analysed in the empirical part. The advertising copies show, for example, a clear increase in the use of "Mitarbeiter" resp. "Mitarbeiterin" ("employee") instead of "kaufm. Angestellte" ("clerk"), "Fabrikarbeiter" ("factory worker"), "Magazingehilfe" ("storeman") or the like. The second part of this article shows how this aspect of language change can be understood through Keller's Invisible-Hand model.
\end{abstract}

\section{$1 \quad$ Vorbemerkungen}

Die Vorstellung, wir würden eine Zeitung aufschlagen und dort folgende Anzeige lesen, lässt uns schmunzeln:

Bei einer Herrschaft in Schaffhausen

findet eine gut empfohlene

Kammerjungfer

dauernde Stelle.

Offerten beliebe man mit Zeugnis-

abschriften und Photographie unter

Chiffre $\S$ einzusenden an

Rudolf Mosse, Schaffhausen.

Diese Stellenanzeige ist im Jahre 1904 in der "Neuen Zürcher Zeitung" erschienen. Sie ist in dieser Form heute undenkbar. Warum kann man das nicht mehr schreiben? Was hat sich geändert? 
Zum einen hat sich die Welt seit Beginn des 20. Jahrhunderts verändert. So schreiben wir heute unsere Zeugnisse nicht mehr ab ("Zeugnisabschrift"), wenn wir uns für eine Arbeitsstelle bewerben, sondern machen eine Fotokopie. Ein Bewerbungsdossier enthält heute "Kopien" sämtlicher Zeugnisse und Berufs- oder Studienabschlüsse sowie ein aktuelles "Foto". Und in einer Stellenanzeige werden wir in der Regel dazu aufgefordert, unsere "Bewerbung" einzusenden, nicht eine "Offerte". Zum anderen hat sich also auch unsere Sprache seit 1904 verändert. Veränderungen in Sprachen in der Zeit werden als "Sprachwandel" bezeichnet. Jede natürliche Sprache ist dem Prozess des Wandels unterworfen, und Sprachwandel erfasst alle Ebenen eines Sprachsystems (Phonologie, Morphologie, Syntax, Semantik), aber auch die Verwendungsbedingungen von Sprache (Sprachgebrauch) (cf. Glück 2000: 675; Schmidt 2000: 20).

Warum verändert sich Sprache? Wie kommt Sprachwandel zustande, und wie geht er vor sich? - Eine vorschnelle Antwort auf diese Frage lautet: "Die Welt ändert sich; es gibt ständig technischen Fortschritt und die Sprache muss sich deshalb ebenfalls ändern, um mit der Entwicklung der Welt Schritt zu halten" (Keller 2000: 4). Bei genauerer Betrachtung hingegen erweist sich dieses Argument als nicht überzeugend. Welche Veränderungen in unserer Welt sollen es denn gewesen sein, die den Wandel von "Offerte" zu "Bewerbung" und von "Photographie" zu "Foto" notwendig gemacht haben? (cf. Keller 1994: 20). Veränderungen in unserer Welt sind weder notwendig noch hinreichend für Veränderungen in unserer Sprache, denn Sprache hat nicht nur die Aufgabe, die Welt abzubilden (cf. Keller 1994: 20). Was ist also verantwortlich für den Wandel? Rudi Keller (1994) geht wie viele andere davon aus, dass Sprachwandel prinzipiell erforschbar und erklärbar ist. Im Einzelnen werden dazu jedoch recht unterschiedliche Positionen vertreten. Die verschiedenen Standpunkte hängen mit grundsätzlichen sprachtheoretischen Auffassungen zusammen ("Was ist Sprache?"; "Was ist die Aufgabe von Sprache?") (cf. Schmidt 2000: 25f.). Auch Keller, dessen Theorie in dieser Arbeit vorgestellt und diskutiert wird, schafft in seiner Sprachwandeltheorie einen eigenen Sprachbegriff.

Im vorliegenden Beitrag wird das Ziel verfolgt, einen neueren Ansatz zur Erklärung von Sprachwandel vorzustellen und eine Auswahl von Reaktionen auf diesen Ansatz auszuwerten. Dazu werden zunächst die wichtigsten Thesen aus Kellers Buch "Sprachwandel: von der unsichtbaren Hand in der Sprache" (1994) resümiert, um die in Abschnitt 3 folgende positive und negative Kritik nachvollziehbar zu machen. Diese kommentierte Übersicht umfasst eine Auswahl kritischer Stimmen, d.h. Buchrezensionen und andere Beiträge, die sich auf Kellers Buchveröffentlichungen aus den Jahren 1990 und 1994 (1. und 2. Auflage von "Sprachwandel") beziehen, sowie die eigene Kritik. Im vierten Abschnitt schliesslich wird anhand eines Beispiels von sprachlicher Veränderung in Stellenanzeigen aus den Jahren 1950-1999 aufgezeigt, dass Kellers Theorie in der empirischen Analyse tatsächlich angewendet werden kann. Mit diesem Vorgehen begegne ich dem häufigen Kritikpunkt, dass nur sehr wenige konkrete Beispiele für Kellers Erklärung von Sprachwandelphänomenen vorliegen. 


\section{Von der unsichtbaren Hand in der Sprache}

Die folgende Gliederung entspricht im Wesentlichen dem Vorgehen Kellers in seinem Buch "Sprachwandel: von der unsichtbaren Hand in der Sprache" (1994) und soll seine Gedankenschritte in Kürze nachzeichnen. Das Buch von Keller ist in zwei Teile mit je drei Kapiteln gegliedert. Während Keller im ersten Teil die Problemstellung, die Ausgangsfragen und erste Grundannahmen thematisiert, entwirft er im zweiten Teil seine "Theorie von der unsichtbaren Hand in der Sprache", setzt sie dann in Bezug zu anderen Sprachwandeltheorien und erklärt Sprachwandel schliesslich zu einem evolutionären Prozess.

\subsection{Darstellung des Problems}

Keller zeigt im ersten Kapitel seines Buches an Beispielen auf, dass Sprachen in permanentem Wandel begriffen sind. Es genügt, eine Zeitung aufzuschlagen, die vor 50 Jahren gedruckt wurde, und wir finden eine Menge von Ausdrücken und Ausdrucksweisen, die heute im gleichen Kontext undenkbar wären (cf. Keller 1994: 18f.). Warum ist das so? Warum verändert sich die Sprache?

Im Folgenden erklärt Keller (1994: 23-25), dass die "falschen" Fragen in Bezug auf den Wandel der Sprache zu unangemessenen Antworten einladen und schliesslich in die Irre führen. Stellen wir die Frage "Warum ändert sich die Sprache?", so präsupponieren wir "Die Sprache ändert sich", obwohl wir wissen, dass es nicht die Sprache ist, die etwas tut, wenn sie sich verändert. Diese Redeweise ist zu hypostasierend. Sie verfährt so, als wäre die Sprache "ein Ding mit ihm innewohnenden Lebenskräften, ein Organismus, wie man im 19. Jahrhundert zu sagen pflegte" (Keller 1994: 25). Fragen wir "Warum ändern die SprecherInnen ihre Sprache?", klingt es zu aktiv, zu geplant, als würden die SprecherInnen ihre Sprache willentlich ändern. So, als wäre die Sprache ein von Menschen gemachtes Artefakt, das sie herzustellen und umzubauen imstande wären.

Beide Rede- oder besser Denkweisen - die erste kann als organistische, die zweite als mechanistische Version bezeichnet werden - sind laut Keller (1994: 25) als Modelle für die einem permanenten Wandel unterliegende Sprache ungeeignet. Kellers These lautet: Die permanente Veränderung unserer Sprache erzeugen wir durch das tägliche millionenfache Benutzen unserer Sprache (cf. Keller 1994: 30). Diese Veränderungen beabsichtigen wir in der Regel nicht, und meist bemerken wir sie auch gar nicht. Die "korrekten" Ausgangsfragen lauten also: "Wieso erzeugen wir durch unser tägliches Kommunizieren einen Wandel? Welches sind die Mechanismen dieser ständigen Veränderung?" (Keller 1994: 30). Um diese Fragen beantworten zu können, müssen wir wissen, wozu wir Sprache verwenden. Auf die Funktion von Sprache geht der Autor im vierten Kapitel ein.

Keller (1994: 34f.) schliesst das erste Kapitel mit einer Analogie, die zu seiner Theorie hinführt: Er zeigt Bilder einer Fotoserie, auf denen festgehalten ist, wie sich Schaulustige auf einem Platz zu zwei Ringen formieren, um zwei Gruppen von Strassenkünstlern zuzuschauen. Diese Struktur entsteht wie der Wandel der Sprache ohne Plan, ohne Verabredung: Sie 
entsteht spontan. Und sie entsteht aus einer Vielzahl einzelner Handlungen, die nach bestimmten Massgaben gewählt worden sind (cf. Keller 1994: 32f.).

\subsection{Die zentralen Denkfiguren}

Laut Keller können wir das Wesen sozialer Phänomene, also die erwähnten zwei Ringe aus schaulustigen Menschen oder den Wandel der Sprache, nicht verstehen, wenn wir die Logik ihrer Genese nicht verstanden haben. Das zweite Kapitel beginnt deshalb mit einer imaginären Geschichte über den Ursprung der Sprache (cf. Keller 1994: 37-51). Diese Geschichte erzählt von Karlheinz, dem Affenmenschen, und zeigt auf, wie der Übergang vom naturhaften Kommunizieren (Angstschrei) zum intentionalen kommunikativen Akt hätte vonstatten gehen können (cf. Keller 1994: 42).

Mit dem Vorgehen in diesem Kapitel verfolgt Keller zwei Ziele. Er führt das sog. Grice'sche Grundmodell ein, welches definiert, was es heisst zu kommunizieren, und er erläutert im Anschluss daran die zentrale Denkfigur seiner Theorie: das Mandeville'sche Paradox (cf. Keller 1994: 50-57). Dieses besagt, dass "moralisch zu missbilligende Bestrebungen der Individuen auf der Ebene der Gesellschaft durchaus billigenswerte Auswirkungen haben können" (Keller 1994: 56f.). Mandeville (1670-1733) erklärte dieses Paradoxon in seiner sog. "Bienenfabel" und zeigte auf, dass der Wohlstand eines (Bienen-)Volkes nicht Ergebnis der Tugenden seiner Bürger, sondern ihrer Untugenden und Laster wie Faulheit, Korruption und Arbeitsscheu ist. Und Sprache - eine durchaus segensreiche Institution - könnte beispielsweise aus dem Bestreben, "die anderen" übers Ohr zu hauen entstanden sein. In dieser paradoxen Denkfigur liegt also die Erkenntnis, dass es gesellschaftliche Phänomene gibt, "die durch Handlungen der Individuen hervorgebracht werden, ohne von diesen intendiert zu sein" (Keller 1994: 57). Sprache ist aus Kellers Sicht ein solches Phänomen: das Ergebnis menschlichen Handelns, nicht aber die Durchführung eines menschlichen Plans (cf. Keller 1994: 58).

Wie lautet nun der Erklärungsmodus solcher Phänomene? - Es ist nicht möglich, den Prozess zu rekonstruieren, durch den die Fähigkeit zur Kommunikation im menschlichen Sinne erzeugt worden ist. Aber wir können aufzeigen, wie sie hätte erzeugt worden sein können. Eine solche Entstehungsgeschichte nennt Keller (1994: 58f.) mit Verweis auf Dugald Stewart (1753-1828), Philosoph der Schottischen Schule, eine "Conjectural History" (vermutende Geschichte). Vermutende Geschichten von Phänomenen wie dem der Kommunikationsfähigkeit oder des Volkswohlstands beruhen nicht auf historischen Fakten, sondern sind philosophische Untersuchungen.

Die Struktur der Argumentation, wie nun aber das Zustandekommen (Genese), die Natur und das Wesen solcher Phänomene erklärt werden können, geht auf den berühmten Moralphilosophen der Schottischen Schule, Adam Smith (1776), zurück und nennt sich "Invisible-Hand-Erklärung" bzw. "Erklärung mittels der unsichtbaren Hand" (cf. Keller 1994: 60f.). "Eine Invisible-hand-Erklärung ist eine Conjectural History eines Phänomens, das Ergebnis menschlichen Handelns, nicht aber Durchführung eines menschlichen Plans ist" 
(Keller 1994: 61). Auf die genaue Struktur einer Invisible-Hand-Erklärung gehe ich weiter unten ein.

\subsection{Sprache als Phänomen der dritten Art}

Mit dem dritten Kapitel schliesst Keller den ersten Teil seines Buches ab, und hier zeigt er auf, weshalb eine befriedigende Antwort auf die Frage des Sprachwandels bis in die jüngste Zeit nicht gefunden wurde. Salopp formuliert lautet sein Argument: Die Forschung war gefangen "in den Fesseln dichotomischen Denkens" (Keller 2000: 4). Man hatte sich in die Frage "verbissen", ob Sprache bzw. der Wandel der Sprache ein Naturphänomen sei oder ein menschliches Artefakt (Kulturphänomen).

Wie kam es überhaupt, dass die Überlegungen der schottischen Moralphilosophen zu Phänomenen, die Ergebnis menschlichen Handelns sind, nicht aber Durchführung eines menschlichen Plans und zum Erklärungsmodus solcher Phänomene (Conjectural History bzw. die Erklärung mittels der unsichtbaren Hand) "den Sprachwissenschaftlern des 19. und 20. Jahrhunderts weitgehend unbekannt geblieben sind" (Keller 1994: 62)? In seiner Antwort verweist Keller (1994: 63) auf die sehr alte Annahme, dass die Welt fein säuberlich in zwei vollständig disjunkte Gegenstandsbereiche aufteilbar sei, nämlich in den Bereich derjenigen Dinge, die es von Natur aus gibt, und den Bereich derjenigen, die künstlich, also vom Menschen gemacht sind. Nicht nur die Dichotomie "Natur versus Kunst" (auf der Ebene der Dinge), sondern auch "Instinkt versus Vernunft" bzw. "Gefühl versus Verstand" (auf der Ebene des Verhaltens) haben sich für das Verständnis dessen, was Sprache ist, als hinderlich erwiesen.

Keller (1994: 64) zeigt, dass zwischen "vernunftgeleitetem" Verhalten und "instinkt- oder gefühlgeleitetem" Verhalten noch eine dritte menschliche Fähigkeit seinen Platz erhalten muss: die Fähigkeit zu regelgeleitetem Verhalten. Wenn ich einen deutschen Satz korrekt bilde, dann folge ich weder meiner Vernunft noch meinem Instinkt, sondern Traditionen, die sich hierzulande herausgebildet haben; ich folge sozialen Regeln. Zwischen Instinkt und Vernunft steht also die Tradition bzw. der Brauch (cf. Keller 1994: 65). Keller (1994: 69) bezeichnet Sprache als einen Brauch - als gigantischen Brauch, um bestimmte Dinge zu bewirken.

Auch zwischen "natürlichen" und "künstlichen" Dingen ist Platz für ein Drittes. In diesem Zusammenhang fasst Keller (1994: 72-81) die wissenschaftliche Sicht von Sprache und Sprachwandel im 19. Jahrhundert zusammen: August Schleicher (1863) betrachtete Sprachen als "Naturorganismen", deren Entwicklung vom Menschen nicht bestimmbar sei, und somit war die Wissenschaft derselben eine Naturwissenschaft. William D. Whitney hingegen vertrat 1874 die Ansicht, dass Sprache eine menschliche Einrichtung, also "von Menschen gemacht" und auch durch sie veränderbar sei. Die Sprachwissenschaft gehöre zu den historischen oder den Geisteswissenschaften. Kellers Fazit aber lautet: Natürliche Sprachen lassen sich weder als Naturphänomene noch als Kulturphänomene zureichend beschreiben. Warum? 
Keller (1994: 83) befindet die Dichotomie "Naturphänomen versus Kulturphänomen" als zu eng für den Sprachbegriff. Die dichotome Sicht der Dinge beruht auf einer unerkannten Zweideutigkeit des Prädikats "von Menschen gemacht". Aus dieser Einsicht entwickelt Keller eine genauere Differenzierung der Dinge und setzt der dichotomen Perspektive eine Trichotomie entgegen. Es gibt Dinge, Keller nennt sie "Phänomene der dritten Art", die weder Natur- noch Kulturphänomene sind, jedoch mit beiden etwas gemeinsam haben. Phänomene der dritten Art sind Ergebnis menschlicher Handlungen (wie die Kulturphänomene), nicht aber Ziel menschlicher Intention (wie die Naturphänomene). Beispiele hierfür sind: ein Autostau "aus dem Nichts"; ein Trampelpfad über den Rasen (cf. Keller 1994: 84f.).

Kellers Hauptthesen am Schluss des ersten Teils seines Buches lauten: Natürliche Sprachen sind Phänomene der dritten Art, und die Betrachtung der Sprache als Phänomen der dritten Art schafft einen Sprachbegriff, "der dem ewigen Wandel der Sprache gerecht wird" (Keller 1994: 85).

\subsection{Die Theorie von der unsichtbaren Hand in der Sprache}

Das vierte Kapitel, und damit der zweite Teil des Buches, beginnt mit einer Zusammenfassung der dargelegten Problemstellung und der Grundannahmen (cf. Keller 1994: 87ff.). Dann erfasst Keller mittels verschiedener Analogien die wesentlichen Eigenschaften von Phänomenen der dritten Art und illustriert so seine Theorie. Ich zeige dies hier an dem viel zitierten Trampelpfad-Beispiel auf: Ein Trampelpfad entsteht durch Handlungen vieler einzelner (viele Personen gehen über eine bestimmte Stelle des Rasens). Er ist also ein kollektives Phänomen. Die das Phänomen erzeugenden Handlungen weisen gewisse Gleichförmigkeiten auf (Personen, die über den Rasen gehen, handeln nach der Maxime: "Geh so von A nach B, dass die gewählte Strecke möglichst kurz ist."). Diese Gleichförmigkeiten mögen für sich genommen irrelevant sein, in ihrer Vielfalt zeitigen sie jedoch bestimmte nicht-intendierte kausale Konsequenzen (Rasenstellen, über die häufig gegangen wird, verkümmern) (cf. Keller 1994: 90). Phänomene der dritten Art, wie der Trampelpfad eines ist, sind immer zusammengesetzt aus einem Mikrobereich (die an der Erzeugung des Phänomens beteiligten Personen bzw. ihre Handlungen), der intentional ist, und einem Makrobereich, der kausaler Natur ist (die durch den Mikrobereich hervorgebrachte Struktur, hier der Trampelpfad) (cf. Keller 1994: 92). Ein Phänomen der dritten Art kann definiert werden als "die kausale Konsequenz einer Vielzahl individueller intentionaler Handlungen, die mindestens partiell ähnlichen Intentionen dienen" (Keller 1994: 92). Es ist von Menschen, ohne dass sie dies beabsichtigt oder auch nur gemerkt hätten, "wie von unsichtbarer Hand geleitet", erzeugt worden.

Wie sieht nun die Struktur einer Erklärung mittels der unsichtbaren Hand oder, wie Keller sagt, einer "Invisible-Hand-Theorie" aus? - Eine Erklärung einer zweischichtigen sozialen Institution wie die der Sprache oder ihres Wandels besteht darin, dass die Makroebene der Institution von der Mikroebene des sozialen Handelns der Individuen hergeleitet wird (cf. Keller 1994: 98). Dies leistet die Invisible-Hand-Theorie über drei Stufen oder anders formuliert: 
Ein Sprachwandelphänomen lässt sich erklären, indem man 1. die Handlungsmotive, -intentionen und -ziele der Individuen benennt sowie die ökologischen (Rahmen-) Bedingungen ihres Handelns (soziale, historische und sprachliche Fakten, welche die SprecherInnen einer Sprache dazu motivieren, ihre Redeweise zu modifizieren), 2. den Prozess darstellt, wie aus der Vielzahl der individuellen Handlungen die zu erklärende Struktur entsteht, und 3. die durch diese Handlungen hervorgebrachte neue Struktur darstellt, bzw. benennt (cf. Keller 1994: 99).

Keller veranschaulicht diesen Prozess anhand zweier Beispiele: der Pejorisierung von "Weib", "Frau" u.a. (cf. Keller 1994: 107-109) und dem Verschwinden von "englisch" (engelhaft) zugunsten von "englisch" (britisch) im 19. Jahrhundert (cf. Keller 1994: 129-131; 113ff.). Zum ersten Beispiel: In unserer Gesellschaft gibt es ein Galanteriegebot Frauen gegenüber. Teil dieses Gebots ist es, beim Reden über Frauen oder Frauen gegenüber Ausdrücke zu wählen, die eher einer höheren Stil- oder Sozialebene angehören als einer niedrigeren. Der Leitsatz (unten "Maxime" genannt) unseres kommunikativen Handelns, wenn das Galanteriespiel gespielt wird, lautet: "Greife bei der Anrede einer Frau lieber eine Etage zu hoch als eine zu niedrig." Dies führt gemäss Keller "mit der Zeit dazu, dass immer tendenziell das 'nächsthöhere' Wort zum unmarkierten Normalausdruck wird, während das ehedem normale pejorisiert wird" (Keller 1994: 108).

Welche anderen Maximen, Motive und Regeln unseres kommunikativen Handelns setzen Invisible-Hand-Prozesse in Gang, an deren Ende die zu erklärenden Strukturen, d.h. die Sprachwandelphänomene, stehen? - Mittels sog. "Handlungsmaximen" erfasst Keller (1994: 126) den Aspekt der relativen Ähnlichkeit des Handelns, welche eine notwendige Bedingung für das Entstehen von Invisible-Hand-Prozessen ist.

Die Hypermaxime unseres Kommunizierens lautet: "Rede so, dass Du sozial erfolgreich bist." Denn gemäss Keller hat eine natürliche Sprache vor allem die Funktion der Beeinflussung der Mitmenschen. Kommunizieren ist somit eine artspezifische Methode, den anderen zu etwas Bestimmtem zu bringen und dient dazu, sozialen Erfolg zu erringen. "Sozial erfolgreich sein" meint hier Einfluss haben, Aufmerksamkeit erhalten, Zuneigung/Nahrungsmittel bekommen, verstanden werden, akzeptiert werden, gelesen werden etc. (cf. Keller 1994: 118-124; 208). Ausformuliert lautet die Hypermaxime: "Rede so, dass Du die Ziele, die Du mit Deiner kommunikativen Unternehmung verfolgst, am ehesten erreichst" (cf. Keller 1994: 142).

Die Hypermaxime kann aufgefächert werden in Untermaximen. Keller (1994: 131-143) unterscheidet dabei (Handlungs-)Maximen, die Veränderung erzeugen (dynamische) und solche, die stabilisierend wirken (statische). Statische Maximen sind: "Rede so, wie Du denkst, dass der andere reden würde, wenn er an Deiner Statt wäre" (Verständlichkeitsmaxime). Und: "Rede so wie die anderen" (Anpassungsstrategie) (cf. Keller 1994: 136f.).

Wenn viele Individuen unter bestimmten ökologischen Bedingungen nach den folgenden Maximen handeln, dann erzeugen sie sprachliche Veränderungen und letztlich Sprachwandel: "Rede so, dass Du beachtet wirst"; "Rede so, dass Du als nicht zu der Gruppe gehörig erkennbar bist"; "Rede amüsant, witzig etc."; "Rede besonders höflich, schmeichelhaft, 
charmant etc."; "Rede so, dass es Dich nicht unnötige Anstrengung kostet" (Ökonomieprinzip) (cf. Keller 1994: 139). Einige dieser Maximen konfligieren miteinander, sie widersprechen sich. Wollen wir dennoch nach beiden zugleich handeln, müssen wir Kompromisse eingehen. Gültig bleibt jedoch bei allen kommunikativen Handlungen die Hypermaxime und damit auch meistens das Ziel, verstanden zu werden (cf. Keller 1994: 140142).

Die Theorie von der unsichtbaren Hand erklärt also ihr Explanandum, ein Phänomen der dritten Art, als "die kausale Konsequenz individueller intentionaler Handlungen, die mindestens partiell ähnliche Intentionen verwirklichen" (Keller 1994: 100f.) und die unter bestimmten ökologischen Bedingungen und nach bestimmten Handlungsmaximen vollzogen worden sind (cf. Keller 1994: 215; siehe auch Grafik 1 im Anhang). Es liegt ein Zusammenwirken von finalen und kausalen Prozessen vor (cf. Keller 1994: 109-117), d.h. Kellers Theorie enthält einen finalen (intentionalen) Erklärungsteil, wie es für KulturphänomenErklärungen kennzeichnend ist, und sie hat einen kausalen Erklärungsteil, wie es für Naturphänomen-Erklärungen kennzeichnend ist (cf. Keller 1994: 100).

\subsection{Kellers Theorie im Lichte anderer Sprachwandeltheorien und sein Fazit}

Auf die letzten beiden Kapitel von Kellers Buch gehe ich in aller Kürze ein, da sie für das Verständnis seiner Theorie nicht zentral scheinen.

Keller kontrastiert seine Invisible-Hand-Theorie im fünften Kapitel mit anderen Theorien von Sprache und ihrem Wandel, genauer gesagt mit Helmut Lüdtkes Sprachwandelgesetz, mit der Natürlichkeitstheorie, mit Noam Chomskys I-Sprache und der Dreiweltentheorie Karl R. Poppers. Lüdtkes Sprachwandelgesetz beispielsweise diskutiert Keller (1994: 147-154) als ein Beispiel einer Invisible-Hand-Erklärung, die Natürlichkeitstheorie jedoch, und insbesondere den unklar bestimmten Begriff der Natürlichkeit und den Erklärungsanspruch dieser Position, kritisiert er stark und schlägt Verbesserungen resp. Ergänzungen vor (cf. Keller 1994: 155167). Im Zusammenhang mit Chomskys I-Sprache schliesslich konstatiert Keller (1994: 171181; 207), dass sich im Rahmen des Sprachbegriffs von Chomsky die Frage nach dem Sprachwandel nicht einmal sinnvoll formulieren lasse.

Ist Sprachwandel ein Spezialfall soziokultureller Evolution? - Im sechsten Kapitel macht Keller (1994: 195-206) deutlich, dass Sprachwandel die drei Bedingungen erfüllt, um als Beispiel eines evolutionären Prozesses zu gelten: 1. ist Sprachwandel eindeutig nicht teleologisch, d.h. er dient nicht der Erreichung eines vorgegebenen Ziels; 2. ist Sprachwandel eindeutig ein kumulativer Prozess (eine entscheidende Eigenschaft von Phänomenen der dritten Art) und 3. beruht die Dynamik des Prozesses auf einem Zusammenspiel von Variation und Selektion. Diese Bedingung ist weniger offensichtlich erfüllt, doch Keller (1994: 197ff.) zeigt auf, dass auch in der Sprache der Mechanismus von Variation und Selektion wirksam ist.

Zum Schluss fasst Keller (1994: 206-215) seine Hauptthesen zusammen und geht in seinem "Plädoyer für Erklärungsadäquatheit" auf Kritik von Roger Lass ein, der die Erklärungsadäquatheit von Sprachwandeltheorien angezweifelt hatte. Keller vertritt die Ansicht, dass die 
Erklärbarkeit sprachhistorischer Phänomene die Wahl der angemessenen Dimension voraussetze. Dies könne nur ein Strukturebene sein, die sich noch sinnvoll mit der Ebene sprachlichen Handelns korrelieren lasse (cf. Keller 1994: 214). Im Sinne der Invisible-HandTheorie bedeutet dies, dass sprachhistorische Daten "als notwendige unbeabsichtigte Konsequenz individueller Handlungen", die "unter bestimmten ökologischen Bedingungen nach bestimmten Handlungsmaximen vollzogen worden sind", ausgewiesen werden müssen (Keller 1994: 215).

\section{$3 \quad$ Kritik}

Es soll nun der Frage nachgegangen werden, wie Kellers Theorie in der Sprachwissenschaft aufgenommen und beurteilt wurde. Dies geschieht anhand einer Auswahl von Buchrezensionen und anderer kritischer Beiträge, die sich auf Kellers Veröffentlichungen aus den Jahren 1990 und 1994 (1. und 2. Auflage von "Sprachwandel") beziehen, sowie anhand verschiedener Publikationen, in denen Kellers Theorie aufgegriffen, mit empirischen Beispielen erweitert oder mit anderen Theorien verknüpft wird.

\subsection{Positive Reaktionen}

In der Literatur besteht weitgehend Einigkeit im Urteil über Kellers Fähigkeit, den anspruchsvollen Stoff gut verständlich, leicht lesbar und unterhaltsam aufzubereiten (cf. Abraham 1991; Stolz 1991; Adamska-Salaciak 1992; Anttila 1992; Nyman 1994; Croft 1997; Fidalgo 1997; Schrodt 1997; Drumm 1998). Die Lektüre sei, wie Greule (1991) feststellt, ein "intellektuelles Vergnügen, dessen Reiz darin liegt, nicht allein nachzuvollziehen, wie Keller die Erklärung mittels der unsichtbaren Hand auf die Sprache überträgt, sondern auch wie er den speziellen Fall des Sprachwandels mit sozialen, politischen und ökonomischen Prozessen in Verbindung bringt" (Greule 1991: 321). Auch Hermanns (1991) hebt die Art des Keller'schen Denkens hervor und kommt zum Schluss, dass uns sein Buch "aus dem engen Netz der ausgetretenen Trampelpfade linguistischer Gedankengänge" hinausführe (Hermanns 1991: 9). Keller betrachte linguistische Probleme nicht als isoliert von den Problemen anderer Wissenschaften, sondern integriere nützliche und geniale Theorien und Lösungen anderer Disziplinen (wie z.B. jene des Moralphilosophen Adam Smith) in seine Sprachwandeltheorie (cf. Hermanns 1991: 9; Croft 1997: 399).

Stolz (1991) und Croft (1997), Autoren zweier vergleichsweise kritischer Rezensionen, geben eher indirekt Hinweise auf Kellers Verdienste. So sieht Stolz den Beitrag Kellers zur modernen Sprachwandeltheorie darin, dass er auf einen möglichen und längst überfälligen "Ausweg aus dem Dilemma von Natur vs. Kultur, Kausalität vs. Finalität, Erklärbarkeit vs. Vorhersagbarkeit in der diachronen Theoriebildung" hinweise (Stolz 1991: 552). Zu letzterem muss ich ergänzen, dass Kellers Theorie in erster Linie Erklärungsadäquatheit anstrebt und keine Prognosen oder die Vorhersagbarkeit von Sprachwandel erlaubt. Sprachwandel ist nicht prognostizierbar, weil das Erfülltsein der Anfangsbedingungen (die ökologischen Bedin- 
gungen und die Handlungsmaximen) nicht vorhersagbar ist ${ }^{1}$ (cf. Keller 1994: 105). Croft äussert seine Hoffnung, dass durch Kellers Buch folgende Irrtümer anderer Theoretiker "zur letzten Ruhe gebettet werden": "die Verdinglichung von Sprache, welche dazu führe, dass deren Wandel durch "Kräfte" oder "Drift" "erklärt" wird; das Augenmerk auf das Sprachsystem anstatt auf den Sprachgebrauch und die entsprechend gerichteten (teleologischen) Erklärungen von Wandelphänomenen" (Croft 1997: 394; Übersetzung d. V.).

Mit einer einzigen Ausnahme (Abraham 1991) stimmen alle RezensentInnen Kellers Ansatz zu. Die einen äussern dies explizit wie beispielsweise Schrodt (1997), der Kellers Grundthesen aufführt (Sprache als Phänomen der dritten Art; Sprachwandel als notwendige Folge unserer Art und Weise des Sprachgebrauchs) und sie als "weitgehend ausser Frage gestellt" bezeichnet (Schrodt 1997: 473). Anttila (1992: 216) ist derselben Meinung und bestätigt Keller auch bezüglich dessen These, dass die Erklärung mittels der unsichtbaren Hand der diesem Typus von Phänomenen einzig adäquate Erklärungsmodus sei. ${ }^{2}$ Andere scheinen implizit mit den theoretischen Ansichten Kellers übereinzustimmen; sie weisen seine Thesen nicht zurück. ${ }^{3}$ Auch AutorInnen theorieverbindender Ansätze (z.B. Bittner 1995) oder theorievergleichender und weiterführender Beiträge (z.B. Wurzel 1997; Elsen 2001) referieren die Invisible-Hand-Theorie bestätigend.

Kellers Buch und seine Invisible-Hand-Theorie - also "eine echt neue und aufschlussreiche Perspektive für die Betrachtung von Sprachwandel" (Nyman 1994: 231; Übersetzung d. V.) ohne Kurzsichtigkeiten und Unterlassungen? - Da müssen wir genauer hinschauen. Im folgenden Abschnitt werde ich auf die häufigsten und gewichtigsten Kritikpunkte eingehen.

\subsection{Kritische und negative Resonanz}

Mit dem Vorwurf, Keller gehe zu wenig auf die Positionen anderer Sprachwandeltheoretiker ein (cf. auch Croft 1997: 393; Schrodt 1997: 474) und biete "sage und schreibe ein Beispiel" an philologischen Illustrationen seiner Thesen, benennt Abraham (1991: 629) in seiner Rezension gleich zwei häufig angebrachte Kritikpunkte.

Bezüglich des ersteren drückt sich wohl Stolz (1991) am pointiertesten aus, wenn er nach einer Auflistung der (von Keller unterschlagenen) gegenwärtig diskutierten Sprachwandelmodelle behauptet, Keller würde ohne erklärende Worte "einfach über den grössten Teil der einschlägigen Literatur samt ihrer Inhalte hinwegsehen" (Stolz 1991: 553). Da Keller vorwiegend die ältere Fachgeschichte (z.B. Schleicher, Whitney) berücksichtige, sei "eine verlässliche Einordnung des Kellerschen Vorschlags in die heutige sprachwandeltheoretische

\footnotetext{
${ }^{1}$ Erklärungen mittels der unsichtbaren Hand erlauben deshalb allenfalls Prognosen hypothetischer Natur: "Wenn die Leute nach den-und-den Maximen handeln, wird unter den-und-den Rahmenbedingungen die-und-die Struktur entstehen" (Keller 1994: 103). Eine Invisible-Hand-Theorie ist also vor allem von diagnostischem Wert. Sie erklärt nicht "wie es weitergeht", sondern "wie es dazu gekommen ist" (Keller 1994: 104).

2 Neben Schrodt (1997) und Anttila (1992) stimmen auch Hermanns (1991), Adamska-Salaciak (1992), Nyman (1994) und Drumm (1998) Kellers Theorie explizit zu.

3 Implizit bestätigende Reaktionen finden wir bei Greule (1991), Stolz (1991), Croft (1997), Fidalgo (1997) und Polomé (1997).
} 
Fachdiskussion" für den Leser "praktisch unmöglich" geworden. Es bestehe die Gefahr, "dass interessierten Laien und Studenten ein viel zu einseitiges Bild von Sprachwandel(theorie) vermittelt" werde (Stolz 1991: 553). Diesem Kritikpunkt kann ich nicht zustimmen, obwohl ich es durchaus geschätzt hätte, bei der Lektüre noch Einblick in andere, neuere Sprachwandeltheorien zu erhalten. Keller (1994: 11) gibt dann im Vorwort zur zweiten Auflage eine explizite Warnung ab: Er beabsichtige nicht, einen Überblick über existierende Theorien abzugeben. Sich diesen Überblick zu verschaffen, ist also Aufgabe der Lesenden.

Nun zum zweiten Kritikpunkt und m.E. schwerwiegenderen Mangel: Mit dem Beispiel des Verschwindens von "englisch" (engelhaft) im 19. Jahrhundert illustriert Keller (1994: 129131) die Gesamtstruktur einer Invisible-Hand-Erklärung zwar sehr eindrücklich, es bleibt nach dem Lesen seines Buches aber der Wunsch nach mehr ausführlichen Beispielen und mehr empirischer Evidenz. Während eine Mehrzahl der AutorInnen vor allem die kleine Zahl der Beispiele bemängelt (cf. Abraham 1991; Greule 1991; Stolz 1991; von Polenz 1991; Schrodt 1997; Drumm 1998), stellen Croft (1997) und Simon (1998) auch noch deren Relevanz bzw. Richtigkeit in Frage (cf. Croft 1997: 396-398; Simon 1998: 140f.). AdamskaSalaciak (1992: 175) fordert nicht weitere Beispiele des bekannten Typs (lexikalischer/ semantischer Wandel), sondern vollkommen andere, beispielsweise phonologischen oder syntaktischen Wandel betreffende. Sie schliesst mit folgenden Worten, denen m.E. zuzustimmen ist: "(...) if invisible-hand explanation is to be taken as the explanation, not just of language change in general, but also of particular instances thereof, one has the right to expect the evidence for the claim to come from as many different categories of change as possible" (Adamska-Salaciak 1992: 175).

Nun sei noch kurz auf mehrfach genannte, jedoch nicht sehr gewichtige Mängel eingegangen: So wird Kellers "meandering style of presentation" kritisiert (Nyman 1994: 231), aufgrund dessen der Argumentationsfluss manchmal behindert sei (cf. auch Greule 1991: 321; Adamska-Salaciak 1992: 165). Bezüglich Kellers Begrifflichkeiten wird angemerkt, dass "Phänomen der dritten Art" eher schwerfällig sei, es jedoch nicht einfach wäre, einen besseren Begriff vorzuschlagen (cf. Nyman 1994: 241). Von Polenz (1991) bezeichnet "abstrakte und hypostasierende Hilfsbegriffe" wie "Phänomene der dritten Art" und "Invisible-HandProzesse" gar als "fragwürdig und wohl überflüssig" (von Polenz 1991: 69), während andere nur das Geheimnisvolle und Magische am Begriff bzw. Wirken der "unsichtbaren Hand" hervorheben (cf. Simon 1998: 140; Croft 1997: 395; Polomé 1997: 203). Schliesslich geben einige AutorInnen ihrem Unmut darüber Ausdruck, dass Keller (1994: Kap. 4.3/6.2) andere, bisherige Erklärungsstrategien und Theorien für Sprachwandel kurzerhand als inadäquat zurückweist (cf. Abraham 1991: 629; Adamska-Salaciak 1992: 177; Simon 1998: 141).

Diese Kritikpunkte betreffen vorwiegend Kellers Stil. Es gibt aber noch eine Reihe von Einwänden, welche die Invisible-Hand-Theorie direkt bzw. ihren Erklärungsanspruch und ihre Reichweite betreffen. Sie lassen sich zu drei Themenbereichen gruppieren:

Ein erster Vorwurf lautet, Invisible-Hand-Erklärungen würden nur für einen Teil der Sprachwandelphänomene zutreffen (cf. von Polenz 1991: 69; Mattheier 1998: 826). Genauer äussert sich dazu Wurzel (1997), der feststellt, dass das Konzept der "unsichtbaren Hand" 
zwar jeden Sprachwandel erfassen wolle, es sich faktisch jedoch auf lexikalischen Wandel beziehe (cf. Wurzel 1997: 302f.; Stolz 1991: 553f.; Elsen 2001: 12). Mattheier (1998: 826) bemängelt, dass Kellers Theorie die lautlichen Bereiche weitgehend ausklammere, und Schrodt (1997: 474) kritisiert, dass Keller keine Antworten auf die "ewigen Fragen" des Sprachwandels, wie z.B. Verbstellung und Lautverschiebung in den germanischen Sprachen, gebe.

Ist Kellers Erklärungsansatz tatsächlich so eingeschränkt? - Das Gegenargument könnte folgendermassen lauten: Wir haben dann eine gute Erklärung für eine sprachliche Veränderung, wenn es gelingt, das zu erklärende Sprachwandelphänomen noch sinnvoll mit der Ebene sprachlichen Handelns zu korrelieren, d.h. plausible Anfangsbedingungen (ökologische Bedingungen, Motive, Handlungsmaximen) zu nennen und einen Invisible-Hand-Prozess, der zwingend daraus folgt, zu schildern (cf. auch Adamska-Salaciak 1992: 166f.) - aus welchem Bereich der Sprache das Phänomen auch stammen mag. Am einfachsten erreicht man dies laut Adamska-Salaciak (1992: 176) im Falle relativ frischen Wandels, der mindestens teilweise semantisch motiviert ist. Ob dies so ist, müssen weitere Untersuchungen, in denen Kellers vorgeschlagene Sprachwandeltheorie angewandt wird, zeigen (cf. z.B. Dürscheid 2000: Erklärung von Veränderungen im Schriftsystem, d.h. "BinnenGrossschreibung" und "Komposita Getrenntschreibung"; Haspelmath 2002: Erklärung der Grammatikalisierung).

Die zweite Einschränkung betrifft die Tatsache, dass Keller sich mehr auf die Verbreitung eines Wandels bezieht als auf die Entstehung eines bestimmten Wandels (cf. Schrodt 1997: 474). Croft (1997: 398f.) kommt zum Schluss, dass die Invisible-Hand-Erklärung nur für die Verbreitung ("propagation") einer neuen Variante gelte; denn in einer Invisible-HandErklärung würden die bereits "kreierten" neuen Varianten als Teil der ökologischen Bedingungen (für die Verbreitung) betrachtet. Croft sieht in der Tatsache, dass Kellers Modell nicht zwischen (unterschiedlichen Mechanismen für) Innovation ("actuation") und Verbreitung ("propagation") eines Wandels unterscheidet, die Hauptschwäche dieses Ansatzes. Er propagiert gar, Kellers "simple model of the collective execution of linguistic innovations, leading to a language change" (Croft 1997: 399) müsse abgeändert werden.

Nun zum dritten und letzten Kritikpunkt. Baldinger (1993: 1), Ronneberger-Sibold (1997: 260) und Schmidt (2000: 26f.) weisen unabhängig voneinander darauf hin, dass Sprachbenutzer wesentlich kreativer, bewusster und outputorientierter handeln ("Sprache mit Absicht verändern"), als Keller es annehme, wenn er vertritt, dass sprachliche Veränderungen eine unbeabsichtigte Folge individueller intentionaler Handlungen seien. Wurzel (1997) liefert das Gegenargument und vertritt damit bestimmt die Meinung Kellers, wenn er schreibt: "Auch wenn die Individuen Handlungsmaximen bewusst verfolgen, absichtsvoll neue sprachliche Mittel auswählen, so tun sie das einzig und allein, um kommunikativ erfolgreich und effektiv zu sein und nicht, um ihre Sprache zu verändern" (Wurzel 1997: 300) (cf. auch Keller 1994: 128f.). Die Frage nach den Motiven individuellen Handelns muss m.E. klar getrennt werden von der Frage nach den Auswirkungen dieses Handelns (cf. auch Keller 1994: 57). 
Neben oben erwähnten Kritikpunkten findet sich eine Reihe anderer Einwände und Ergänzungen, alles Einzelnennungen, auf die an dieser Stelle nicht eingegangen werden kann. Diesbezüglich besonders ausführliche und lesenswerte Rezensionen sind jene von Stolz (1991), Adamska-Salaciak (1992), Nyman (1994) und Croft (1997).

\subsection{Fazit}

Die hier vorgetragene Auswertung einer Auswahl von Buchrezensionen und anderen kritischen Beiträgen zu "Sprachwandel" (1. und 2. Auflage) zeigt, dass Kellers Veröffentlichung viel Beachtung erfahren hat. Die positive Resonanz überwiegt die kritische oder gar negative (cf. auch Keller 1994: 11). Schwerwiegende Mängel sind nicht vorhanden, oder mit den Worten Anttilas (1992): "Dieses Buch ist bemerkenswert gut; ich konnte keinen echten Fehler finden. Es wird einigen Aufwand erfordern, es zu übertreffen" (Anttila 1992: 218; Übersetzung d. V.).

Die beiden gewichtigsten Vorwürfe - der eine betrifft die kleine Zahl der Beispiele, welche die Struktur einer Invisible-Hand-Erklärung aufzeigen und beweisen, dass Sprachwandelphänomene mittels Kellers Theorie adäquat erklärt werden können, der andere betrifft den Kritikpunkt, Invisible-Hand-Erklärungen würden nur für einen Teil der Sprachwandelphänomene zutreffen - lassen sich entkräften. Man kann, wie beispielsweise Bittner (1995: 120f.), die Invisible-Hand-Theorie mit einem anderen Sprachwandelkonzept (Konzept des natürlichen grammatischen Wandels) verbinden, um zu einem neuen Modell zur Erklärung sprachlichen Wandels zu gelangen, das zudem verschiedene Wandeltypen unterscheidet und beschreibt. Bittner legt auch ein Beispiel morphologischen Wandels vor (Übergang des im Mhd. starken Verbes smiegen - smouc zum schwachen nhd. Verb schmiegen), dessen Erklärungsstruktur einer Invisible-Hand-Erklärung sehr ähnlich ist. Mit diesem Vorgehen erweitert er die Palette von Sprachwandelphänomenen, die mittels des Invisible-Hand-Konzepts erklärt werden können.

Weitere Beispiele von Invisible-Hand-Erklärungen sind bei Dürscheid (2000) und Haspelmath (2002) zu finden. Die Liste der in der Literatur gegebenen Beispiele bleibt allerdings relativ kurz. Ich will deshalb im folgenden Abschnitt versuchen, ein weiteres beizufügen.

\section{$4 \quad$ Sprachwandel in Stellenanzeigen}

Sprachwandel gibt es in allen Sprachen, die in aktivem Gebrauch sind, zu allen Zeiten, und er vollzieht sich auf allen sprachlichen Ebenen (cf. Keller 2000: 1). Gelegentlich werden wir ZeugInnen aktuellen Sprachwandels, etwa wenn uns im Alltag die zunehmende "BinnenGrossschreibung" bei Substantiven (z.B. BauBedarf, ProSieben) auffällt, oder wir werden uns des Wandels bewusst, wenn wir alten Menschen beim Sprechen zuhören und sie Ausdrücke oder Redewendungen gebrauchen, die wir nicht mehr in unserem Repertoire haben. Viele Sprachwandelprozesse aber, z.B. Grammatikalisierung, sind nicht etwas, das von heute auf morgen geschieht oder von einer Generation zur nächsten. Jede Generation trägt zwar "ihren Teil zu den grossen Schwingungen des Rades der Sprachgeschichte bei, aber für 
die einzelnen Sprecher sind diese Veränderungen praktisch nicht wahrnehmbar" (Haspelmath 2002: 271).

Wahrnehmbar werden sprachliche Veränderungen jedoch durch schriftliche und mündliche Sprachquellen aus verschiedenen Jahrzehnten oder Jahrhunderten, wie etwa Briefe, Radiosendungen oder Anzeigen. Eben dies ist Gegenstand des nächsten Abschnitts: die sprachlichen Veränderungen in (Stellen-)Anzeigen.

\subsection{Sprachliche Aufwertung im Bereich von Berufsbezeichnungen}

Im Folgenden werde ich ein Beispiel eines lexikalischen Wandels genauer analysieren und die sprachliche Veränderung mit dem dreistufigen Modell einer "Invisible-Hand-Erklärung" zu erfassen versuchen. Zum lexikalischen Sprachwandel in der Nachkriegszeit schreibt von Polenz (1999) folgendes: "Eine für das 20. Jahrhundert typische Art von Wortersatz durch Neologismen ist der Bezeichnungswandel zur 'sozialen Aufwertung' von Berufen und anderen Personenstandsbegriffen mit geringerem Sozialprestige (...), auch 'soziale Euphemismen', 'Schonungswörter' (...) genannt" (von Polenz 1999: 380). Hier interessiert insbesondere die Aufwertung von Berufsbezeichnungen. Ein bekanntes und häufig verwendetes Beispiel dafür ist die "Putzfrau", die zur "Raumpflegerin" wurde. Die Reihe der Berufsaufwertungen habe schon um 1900 begonnen, erklärt von Polenz, "als sich Dienstbotenvereine um die Abschaffung von Gesinde, Knecht, Magd, Diener, Dienstmädchen, ... bemühten, wobei das Letztgenannte in mehreren Ersatzstufen durch Stütze, Hauspersonal, Haushaltshilfe, Reinigungskraft, Hausangestellte, Raumpflegerin usw. verdrängt wurde" (von Polenz 1999: 380). Zur Sprachmode wurde die Berufsaufwertung dann in der Zeit des Wirtschaftswunders und der Sozialharmonisierung in der Nachkriegszeit. Es lässt sich in diesem Zusammenhang auch ein Trend zu mehr Komposita statt Ableitungen, mit möglichst abstrakten Bereichs- statt Tätigkeitsbezeichnungen (z.B. -angestellter, -mitarbeiter, -assistent, -(fach)kraft, -experte, -spezialist, -techniker, -berater etc., auch mit "Hochwert-Präfixoiden" wie Ober-, Super-, Top- und Spitzen-) feststellen (cf. von Polenz 1999: 380). Die Prestigeerhöhung durch den Begriff "weibliche Verkaufskraft" gegenüber der herkömmlichen lexikalisierten Bildung "Verkäuferin" (cf. Ortner 1999: 339) oder "Top-Verkäufer" anstatt "Verkäufer" sind konkrete Beispiele hierfür.

Ortner (1999) arbeitet in ihrer Untersuchung von 3547 Stellenanzeigen in österreichischen Tageszeitungen des 20. Jahrhunderts heraus, dass es in neuester Zeit eine Tendenz zur Verwendung von "Schmeichelwörtern" wie Profi, Talent oder Persönlichkeit gebe (cf. Ortner 1999: 338; siehe Grafik 2 im Anhang). Die Textsorte "Stellenanzeige" eignet sich m.E. sehr gut, um sprachliche Veränderungen dieser Art zu beobachten und empirisch nachzuweisen. Dies wurde verschiedentlich auch schon gemacht. So hat beispielsweise Oksaar (1976) Sprachwandelprozesse unter dem Gesichtspunkt der Umwertung (Auf- und Abwertung) im Bereich der Berufs- und Tätigkeitsbezeichnungen u.a. in Stellenanzeigen untersucht. Spätere Studien, z.B. jene von Wittemöller (1988) und Ortner (1999), befassen sich schwerpunktmässig mit der Wortbildung und dem Gebrauch von (weiblichen) Berufsbezeichnungen, berücksichtigen also geschlechtsspezifische Aspekte (bspw. den Übergang von der 
geschlechtsspezifischen Bezeichnung zur Doppelnennung oder geschlechtsneutralen Bezeichnung: Stenotypistin > Sekretär/in oder Organisationstalent, cf. Ortner 1999: 327).

Bendel (2000) schliesslich untersucht mittels einer Inhaltsanalyse von Stellenanzeigen aus Schweizer Tageszeitungen (1950-1994) nicht sprachliche Veränderungen bei Berufsbezeichnungen, sondern den "Wandel der Anzeigensprache" und damit den Wandel der Textsorte Stelleninserat. Ich werde für meine kleine Untersuchung denselben Datensatz verwenden. Dabei handelt es sich um eine Stichprobe von 26`195 Stellenanzeigen, die zwischen 1950 und 1999 in insgesamt etwa 70 Zeitungen und Anzeigern der Deutschschweiz erschienen sind. Die Erstellung des repräsentativen Datensatzes erfolgte mittels eines mehrstufigen Auswahlverfahrens, unter Berücksichtigung des bestmöglichen Zeitpunktes sowie einer repräsentativen Vertretung der verschiedenen Regionen und Wirtschaftssektoren der Deutschschweiz im Zeitverlauf. Diese Daten wurden im Rahmen des Forschungsprojektes "Langfristiger Wandel der Qualifikationsnachfrage auf dem Stellenmarkt 1950-2002" unter der Leitung von Prof. Dr. Marlis Buchmann und Dr. Stefan Sacchi, Universität und ETH Zürich, erhoben (siehe auch im Internet unter www.arbeitsmarktforschung.ch).

\subsection{Wenn der "Magazingehilfe" zum "Mitarbeiter" wird}

Betrachten wir Stellenanzeigen aus den Jahren 1950 bis 1999, können wir Veränderungen auf allen sprachlichen Ebenen feststellen. Nehmen wir nur die durch Fettschrift und Zeilenabstände grafisch hervorgehobene Berufs- oder Personenbezeichnung ins Visier ("das, was mittels Anzeige gesucht wird"), fällt folgendes auf: Einzelne Bezeichnungen verschwinden im Laufe der Zeit (z.B. Melker, Knecht, Jüngling, Tochter). Neue Bezeichnungen tauchen, auch im Zusammenhang mit neuen Berufen und neuen Tätigkeitsfeldern, auf (z.B. Product-Manager, Call-Center-Agent, Software-Entwickler). Und es kommt vor, dass alte Bezeichnungen durch neue, "wertvollere" oder "prestigeträchtigere" abgelöst werden (z.B. Vertreter > Aussendienstmitarbeiter; Arztgehilfin > med. Praxisassistentin; Putzfrau > Raumpflegerin, siehe Grafik 3 im Anhang), ohne dass sich die in diesem Beruf ausgeübte Tätigkeit massgeblich verändert hätte.

Ich werde nun der Frage nachgehen, ob sich auch in den vorliegenden Daten der bereits beschriebene Trend der Aufwertung mittels Gebrauch einer möglichst abstrakten Bereichsstatt Tätigkeitsbezeichnung (z.B. "Mitarbeiterin für den Verkauf" anstatt "Verkäuferin") nachweisen lässt (cf. von Polenz 1999: 380). Dies werde ich am Beispiel der Bezeichnung "MitarbeiterIn" tun. Dabei berücksichtige ich alle Fälle, in denen in einer Stellenanzeige nicht die für den Beruf übliche Bezeichnung verwendet wird, sondern diese andere - "mit dem Ziel, die Geltung des Berufes im sozialen Leben und das Individual- und Sozialprestige des Berufsausübers zu erhöhen" (Oksaar 1976: 28). Die statistische Auszählung der Häufigkeit von "Mitarbeiter" resp. "Mitarbeiterin(nen)" als Berufs- oder Personenbezeichnung in den 26 195 Stellenanzeigen ergibt folgendes Bild: 


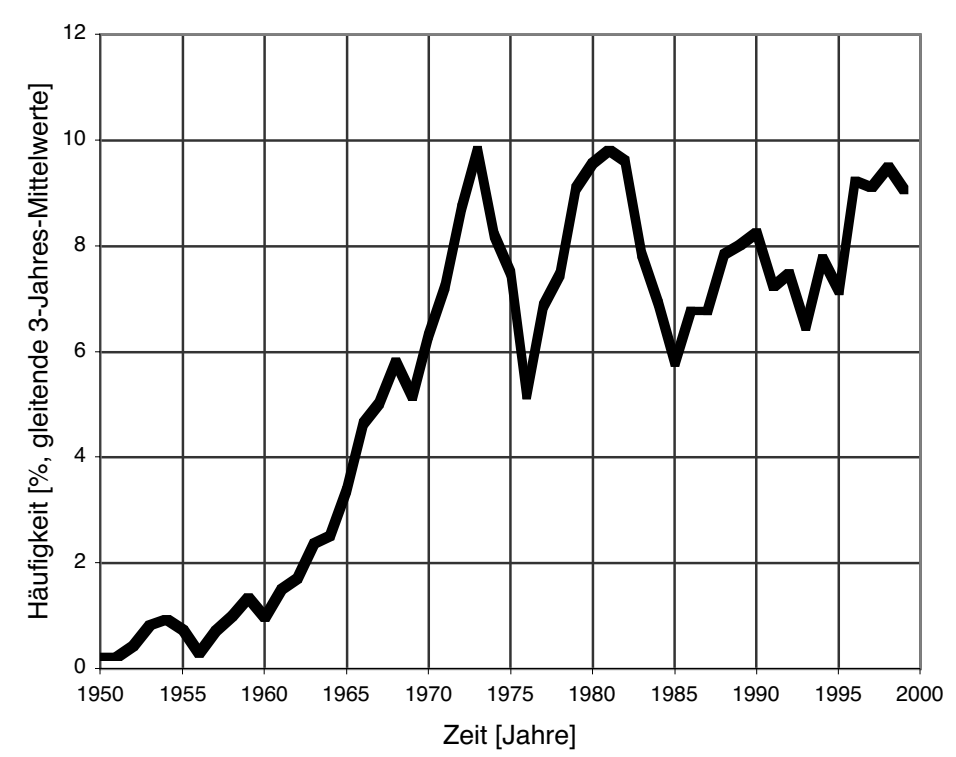

Grafik: Relative Häufigkeit von "Mitarbeiter" resp. "Mitarbeiterin(nen)" als Berufs- oder Personenbezeichnung in Stellenanzeigen 1950-1999. Ausgeschlossen wurden Fälle, in denen das Wort "MitarbeiterIn" schon in der üblichen Berufsbezeichnung (erschlossener Beruf) vorkommt (z.B. Aussendienstmitarbeiter), da hier keine Aufwertung vorliegt.

Diese Grafik zeigt, dass die Verwendungshäufigkeit der Bezeichnung "Mitarbeiter" resp. "Mitarbeiterin(nen)" (anstelle der für den Beruf üblichen Bezeichnung) in Stellenanzeigen zwischen 1956 und 1973 stark angestiegen ist. Es sind drei markante Einbrüche erkennbar (Mitte 70er, Mitte 80er, anfangs 90er Jahre). Insgesamt gesehen gehen die Zahlen nach 1980 wieder leicht zurück, doch bleibt die durchschnittliche Anzahl pro Jahr, verglichen mit den ersten 15 Jahren der beobachteten Zeitspanne, hoch.

Welche Berufe werden hinter der Bezeichnung "Mitarbeiter" resp. "Mitarbeiterin(nen)" "versteckt"? - Wenn wir die Zusammensetzung der erschlossenen Berufe betrachten (siehe Grafik 4 im Anhang), zeigt sich, dass in den ersten zehn Jahren (1950-1960) vor allem gut qualifizierte Angestellte (Bankangestellte, kaufmännische Angestellte etc.) gesucht wurden, wenn von "MitarbeiterInnen" die Rede war. Ab 1955 wurden mit diesem Begriff zusätzlich Arbeiter (Fabrikarbeiterin, Lagerarbeiter etc.), ab 1960 auch unqualifizierte Hilfskräfte (Magazingehilfe, Bürogehilfin, Hilfsmonteur etc.) bezeichnet, Berufe mit tiefem Sozialprestige also. Am häufigsten wurde die Bezeichnung "Mitarbeiter" resp. "Mitarbeiterin(nen)" jedoch für Angestellte verwendet.

Wie kam es dazu, dass diese Bezeichnung einen solchen "Boom" erlebte und dass heute z.B. eine Schuhfabrik eher "MitarbeiterInnen" sucht anstatt "SchuhfabrikarbeiterInnen"? - Diese sprachliche Veränderung lässt sich mit dem dreistufigen Modell einer Invisible-HandErklärung erfassen. Dazu müssen die Motive, Intentionen und Ziele, die den Handlungen der Individuen, die an der Erzeugung des betreffenden Phänomens beteiligt sind, zugrunde liegen, einschliesslich der Rahmenbedingungen ihres Handelns, benannt werden (cf. Keller 1994: 99). Was veranlasst die VerfasserInnen von Stellenanzeigen dazu, "Mitarbeiter" resp. "Mitarbeiterin(nen)" anstatt der korrekten Berufsbezeichnung zu schreiben? - Diese Bezeich- 
nung soll Beachtung finden, denn eine Anzeige will gelesen werden (Handlungsmaxime: "Schreibe so, dass Du beachtet wirst."). Sie soll schmeicheln, aber verständlich bleiben. Der Lesende soll sich angesprochen fühlen und sich beim inserierenden Betrieb melden. Wichtige Rahmenbedingungen, unter denen die Schreibenden handeln, sind jene wirtschaftlichen und sozialen Entwicklungen anfangs der zweiten Hälfte des 20. Jahrhunderts, die auch den Arbeitsmarkt beeinflussten. Dazu gehören u.a. das Wirtschaftswachstum zwischen 1950 und 1975 und die sich verändernde Einstellung zur Arbeit ("vom Ethos der Pflichterfüllung zum Ethos der Selbstverwirklichung"; "von der Arbeits- zur Freizeitgesellschaft", cf. Buchmann und Eisner 1998). Dazu kommt eine sprachliche Bedingung: Die Bezeichnung "Mitarbeiter" ruft die Konnotation hervor, dass ein Arbeitnehmer nicht nur "Angestellter" oder "(Hilfs-) Arbeiter" ist, sondern ein (gleichberechtigter) Mitarbeiter mit mehr Entfaltungsmöglichkeiten und Sozialprestige.

In der Zeit, in der die Wirtschaft blühte, folglich mehr freie Stellen zu besetzen waren, und in der sich "Privatleben und Freizeit als Quellen von Identität gegenüber der Arbeit" emanzipierten (Buchmann und Eisner 1998: 118), wurden die wenigen Stellensuchenden besonders häufig mit "MitarbeiterIn" umworben. In jeder Rezession jedoch gibt es einen Einbruch der Verwendungshäufigkeit dieser Bezeichnung (siehe Grafik).

Die aufwertende Bezeichnung "Mitarbeiter" resp. "Mitarbeiterin(nen)" hat sich infolge einer Vielzahl intentionaler Handlungen einzelner verbreitet, was vermutlich mit der Zeit auch auf eine Anpassungsstrategie zurück geht (Handlungsmaxime: "Schreibe so wie die anderen."). Der leichte Rückgang in den vergangenen Jahren könnte auf eine Art Inflation zurückzuführen sein: Der "billige Griff nach einem 'wertvolleren Ausdruck'" (Haspelmath 2002: 273) (für immer weniger qualifizierte Arbeitskräfte) hat letztlich zum Wertverlust geführt, d.h. die Bezeichnung "Mitarbeiter" resp. "Mitarbeiterin(nen)" ist nicht mehr so viel wert wie früher. Es ist anzunehmen, dass sie heute durch neue, "noch wertvollere" Bezeichnungen ergänzt wird.

Solche Invisible-Hand-Erklärungen sind "vermutende Geschichten" (Conjectural Histories, cf. Keller 1994: 61) von Phänomenen, die Ergebnis menschlichen Handelns, nicht aber Durchführung eines menschlichen Planes sind. Die "Geschichte" darüber, wie die Verbreitung der aufwertenden Bezeichnung "Mitarbeiter" resp. "Mitarbeiterin(nen)" in Stellenanzeigen vor sich gegangen sein könnte, stellt den Versuch einer guten Erklärung dar. Gut ist sie, wenn die Anfangsbedingungen plausibel sind und der Invisible-Hand-Prozess zwingend daraus folgt. Der Wahrheitswert einer Invisible-Hand-Theorie ist gemäss Keller (1994: 102) jedoch meist nicht feststellbar, da die Wahrheit der wesentlichen Anfangsbedingungen (Motive, Handlungsmaximen, ökologische Bedingungen) nicht feststellbar ist. So könne beispielsweise der "Wahrheitswert von Aussagen über Motive, die Handlungsweisen zugrunde liegen, (...) vielfach sowohl aus technischen als auch aus psychologischen Gründen weder verifiziert noch falsifiziert werden" (Keller 1994: 102). In der oben beschriebenen "Geschichte" ist z.B. nicht zu beweisen, ob die Handlungsmaxime "Schreibe so, dass Du beachtet wirst" tatsächlich zur Anwendung gekommen ist. Der beschriebene Invisible-Hand-Prozess ist zudem der direkten Beobachtung entzogen. Dies alles muss jedoch den Erklärungswert einer Invisible-Hand- 
Erklärung nicht schmälern, denn "sie kann gut oder schlecht sein unabhängig von der Feststellbarkeit ihres Wahrheitswertes" (Keller 1994: 102). Eine gute Invisible-HandErklärung erfordert vertiefte Kenntnisse sowohl der Keller`schen Sprachwandeltheorie als auch der pragmatischen Bedingungen und der jeweils herrschenden gesellschaftlichen Verhältnisse. Für eine ausführliche Darstellung des Beispieles wären deshalb disziplinübergreifendes Wissen und weitergehende Untersuchungen notwendig.

\section{$5 \quad$ Zusammenfassung}

Einleitend wurde von der Frage ausgegangen, was denn verantwortlich sein könnte für den Sprachwandel. Kellers Antwort lautete, dass wir die permanente Veränderung unserer Sprache durch das tägliche millionenfache Benutzen der Sprache erzeugen und Sprachwandel ein unbeabsichtigter, unreflektierter "Nebeneffekt" kommunikativen Handelns ist. In der kritischen Betrachtung der Invisible-Hand-Theorie hat sich gezeigt, dass neben viel Lob für Kellers Vorgehen und dessen Denkweise sowie einer breiten Zustimmung hinsichtlich des theoretischen Ansatzes auch berechtigte Einwände vorhanden sind. Diese betreffen vor allem die kleine Zahl der Beispiele, die Keller zur Illustration seiner Invisible-Hand-Erklärung heranzieht, sowie eine gewisse Einschränkung des Erklärungsansatzes auf einen Teil der Sprachwandelphänomene (lexikalischer/semantischer Wandel). Zudem stiess Kellers Stil auf Kritik. Gesamthaft betrachtet hat sein Beitrag zur modernen Sprachwandeltheorie jedoch sehr viel Beachtung erfahren. In einer kleinen Untersuchung von Stellenanzeigen, die zwischen 1950 und 1999 in Schweizer Tageszeitungen erschienen sind, wurde schliesslich eine sprachliche Veränderung, die von Polenz (1999: 380) als typisch für das 20. Jahrhundert bezeichnet hat, empirisch nachgewiesen. Die statistische Auszählung der Verwendungshäufigkeit der aufwertenden Bezeichnung "Mitarbeiter" resp. "Mitarbeiterin(nen)" hat ergeben, dass diese stark angestiegen ist. Diese Verbreitung konnte mittels einer InvisibleHand-Erklärung erfasst werden.

Die Theorie von der unsichtbaren Hand in der Sprache ist m.E. ein faszinierender Ansatz zur Beschreibung und Erklärung von Sprachwandel im Allgemeinen. Ginge es jedoch um die Erklärung einzelner Wandeltypen oder spezieller Sprachwandelphänomene, so dürften die Grenzen spürbar werden. Vielleicht aber ist gerade dies das Reizvolle am Wandel - dass er sich einer lückenlosen und garantiert "wahren" Erklärung oft entzieht.

\section{Literaturangaben}

\section{Sprachwandel und Sprachgeschichte}

Baldinger, Kurt (1993): "Ist die unsichtbare Hand wirklich unsichtbar? Kritische Betrachtungen zum Bedeutungswandel". In: Schmidt-Radefeldt, Jürgen/Harder, Andreas (eds.): Sprachwandel und Sprachgeschichte. Festschrift für Helmut Lüdtke zum 65. Geburtstag. Tübingen: 1-8.

Bittner, Andreas (1995): "Im Schatten der unsichtbaren Hand". In: Boretzky, Norbert et al. (eds.): Natürlichkeitstheorie und Sprachwandel. Bochum: 111-122 (= Bochumer-Essener Beiträge zur Sprachwandelforschung 22). 
Dürscheid, Christa (2000): "Verschriftungstendenzen jenseits der Rechtschreibreform". Zeitschrift für germanistische Linguistik 28/2: 237-247.

Glück, Helmut (ed.) (2000): Metzler-Lexikon Sprache. 2. Auflage. Stuttgart/Weimar.

Elsen, Hilke (2001): "Formen, Konzepte und Faktoren der Sprachveränderung". Zeitschrift für germanistische Linguistik 29/1: 1-22.

Haspelmath, Martin (2002): "Grammatikalisierung: von der Performanz zur Kompetenz ohne angeborene Grammatik". In: Krämer, Sybille/König, Ekkehard (eds.): Gibt es eine Sprache hinter dem Sprechen? Frankfurt am Main: 262-286.

Keller, Rudi (1994): Sprachwandel. Von der unsichtbaren Hand in der Sprache. 2. Auflage. Tübingen/Basel.

Keller, Rudi (2000): Sprachwandel. BDÜ 2000: Faszination Sprache - Herausforderung Übersetzung (unveröffentlichter Aufsatz). Gesehen am 7. November 2003 unter: http://www.phil-fak.uni-duesseldorf.de/rudi.keller/index.php?check_js.php\&1.

Mattheier, Klaus J. (1998): "Allgemeine Aspekte einer Theorie des Sprachwandels". In: Besch, Werner (ed.): Sprachgeschichte. Ein Handbuch zur Geschichte der deutschen Sprache und ihrer Erforschung. Berlin: 824-836. (= Handbücher zur Sprach- und Kommunikationswissenschaft 2).

Polenz, Peter von (1991): Deutsche Sprachgeschichte vom Spätmittelalter bis zur Gegenwart. Einführung; Grundbegriffe; Deutsch in der frühbürgerlichen Zeit. Berlin.

Polenz, Peter von (1999): Deutsche Sprachgeschichte vom Spätmittelalter bis zur Gegenwart. 19. und 20. Jahrhundert. Berlin.

Ronneberger-Sibold, Elke (1997). "Sprachökonomie und Wortschöpfung". In: Birkmann, Thomas et al. (eds.): Vergleichende germanische Philologie und Skandinavistik. Festschrift für Otmar Werner. Tübingen: 249-261.

Schmidt, Wilhelm (ed.) (2000): Geschichte der deutschen Sprache. Ein Lehrbuch für das germanistische Studium. 8. Auflage. Stuttgart/Leipzig.

Simon, Hans-Joachim (1998): Sprachen im Wandel. Frankfurt am Main.

Wurzel, Wolfgang Ullrich (1997): "Natürlicher Grammatischer Wandel, "unsichtbare Hand" und Sprachökonomie - Wollen wir wirklich so Grundverschiedenes?" In: Birkmann, Thomas et al. (eds.): Vergleichende germanische Philologie und Skandinavistik. Festschrift für Otmar Werner. Tübingen: 295-308.

\section{Rezensionen der beiden Auflagen von Kellers "Sprachwandel"}

Abraham, Werner (1991): "Sprachwandel. Von der unsichtbaren Hand in der Sprache" (Keller 1990). Germanistik 32: 628-629.

Adamska-Salaciak, Arleta (1992): "Language Change as a Phenomenon of the Third Kind" (Keller 1990). Folia Linguistica Historica 12/1-2: 159-180.

Anttila, Raimo (1992): "On Language Change. The Invisible Hand in Language" (Keller 1990). Studies in Language 16/1: 213-219.

Croft, William (1997): "On Language Change. The Invisible Hand in Language" (Keller 1994). Journal of Pragmatics 27/3: 393-400. 
Drumm, Daniela (1998): "On Language Change. The Invisible Hand in Language" (Keller 1994). International Review of Applied Linguistics in Language Teaching (IRAL) 36/4: 363-364.

Fidalgo, Reyes I. (1997): "On Language Change. The Invisible Hand in Language" (Keller 1994). Language 73/1: 193.

Greule, Albrecht (1991): "Sprachwandel. Von der unsichtbaren Hand in der Sprache" (Keller 1990). Zeitschrift für Dialektologie und Linguistik 58/3: 319-321.

Hermanns, Fritz (1991): "Wie sich Sprachen wandeln" (Keller 1990). Sprachreport 4: 7-9.

Nyman, Martti (1994): "Review Article. Language Change and the 'Invisible Hand'" (Keller 1990). Diachronica 11/2: 231-258.

Polomé, Edgar C. (1997): "On Language Change. The Invisible Hand in Language" (Keller 1994). The Journal of Indo-European Studies 25/1-2: 202-203.

Schrodt, Richard (1997): "Sprachwandel. Von der unsichtbaren Hand in der Sprache" (Keller 1994). Beiträge zur Geschichte der Deutschen Sprache und Literatur 119/3: 473-474.

Stolz, Thomas (1991): "Sprachwandel. Von der unsichtbaren Hand in der Sprache" (Keller 1990). Zeitschrift für Phonetik, Sprachwissenschaft und Kommunikationsforschung (ZPSK) 44/4: 550-554.

\section{Sprachwandel in Stellenanzeigen}

Bendel, Sylvia (2000): Von der Stellenausschreibung zur Personalwerbung. Sprachliche Veränderungen in den Stelleninseraten und ihre Bedeutung (unveröffentlichter Aufsatz). Soziologisches Institut der Universität Zürich.

Buchmann, Marlis und Eisner, Manuel (1998): "Arbeit und Identität - Von der Notgemeinschaft zur Wahlverwandtschaft". In: Geiser, Thomas et al. (eds.): Arbeit in der Schweiz des 20. Jahrhunderts. Wirtschaftliche, rechtliche und soziale Perspektiven. Bern: 111-134.

Oksaar, Els (1976): Berufsbezeichnungen im heutigen Deutsch. Soziosemantische Untersuchungen. Mit deutschen und schwedischen experimentellen Kontrastierungen. Düsseldorf. (= Schriften des Instituts für deutsche Sprache, Sprache der Gegenwart 25).

Ortner, Lorelies (1999): "Stellenanzeigen und Geschlecht: Sprachwandel in österreichischen Zeitungen des 20. Jahrhunderts". In: Pümpel-Mader, Maria/Schönherr, Beatrix (eds.): Sprache - Kultur - Geschichte. Sprachhistorische Studien zum Deutschen. Innsbruck: 325359. (= Germanistische Reihe 59).

Wittemöller, Regina (1988): Weibliche Berufsbezeichnungen im gegenwärtigen Deutsch. Bundesrepublik Deutschland, Österreich und Schweiz im Vergleich. Frankfurt am Main. (= Europäische Hochschulschriften, Deutsche Sprache und Literatur 1083). 


\section{Anhang}

Grafik 1: Die Struktur einer Invisible-Hand-Erklärung

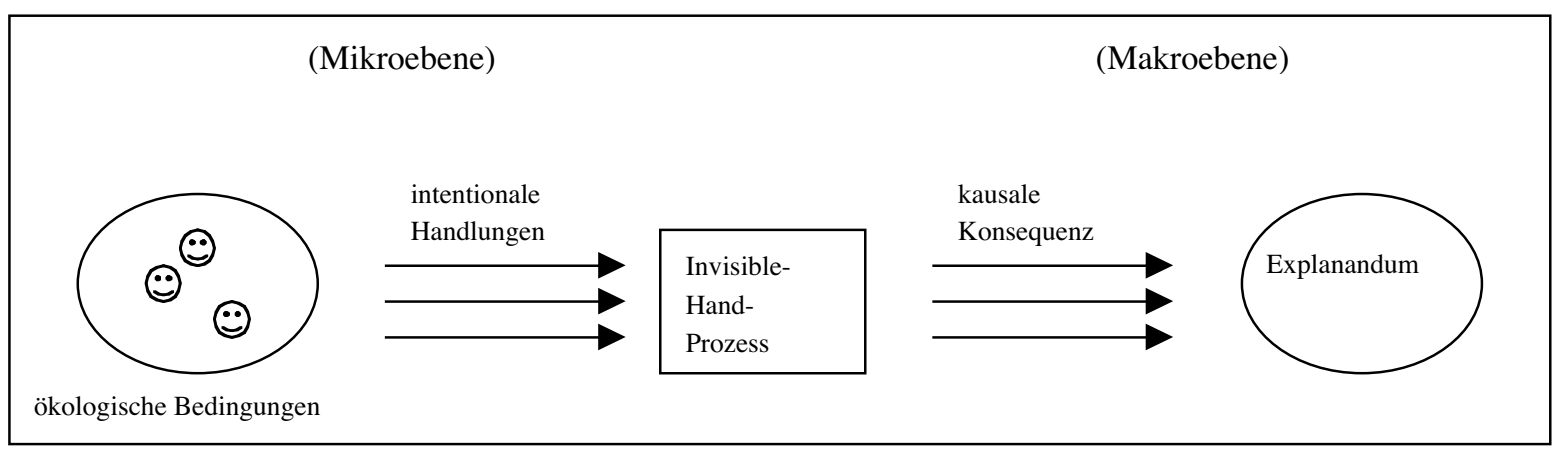

Quelle: Keller 1994: 125

Grafik 2: Die Schmeichelwörter "Profi", "Talent" und "Persönlichkeit"

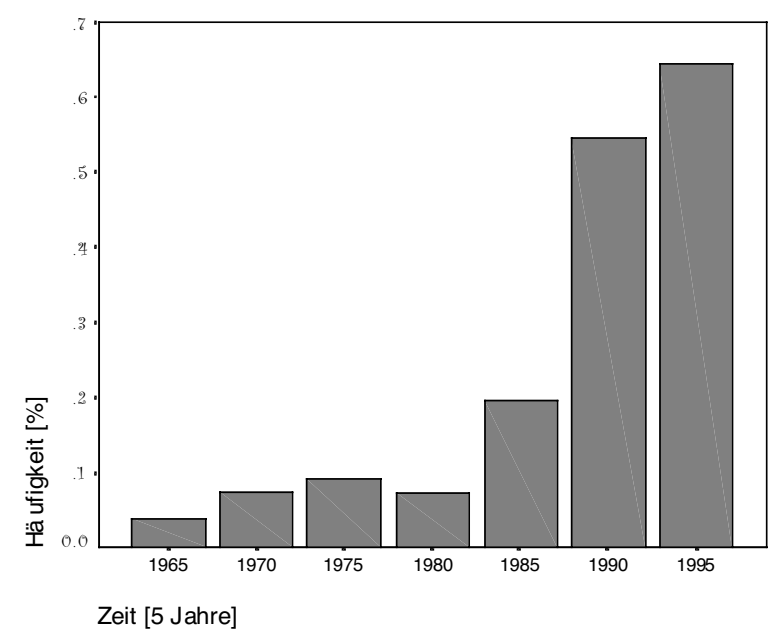

Relative Häufigkeit der Schmeichelwörter "Profi", "Talent" und "Persönlichkeit" in deutschschweizerischen Stellenanzeigen 1950-1999 (anstelle der üblichen Berufsbezeichnung), erste Nennungen ab 1965.

Der Trend, herkömmliche Berufsbezeichnungen durch Komposita mit den Schmeichelwörtern "Profi", "Talent" oder "Persönlichkeit" zu ersetzen, ist vor allem im Berufsfeld "Verkauf" erkennbar (Bsp.: "Profiverkäuferin", "Verkaufstalent" oder "Teilzeitverkaufspersönlichkeit"). 
Grafik 3: Die "Putzfrau" wird zur "Raumpflegerin"

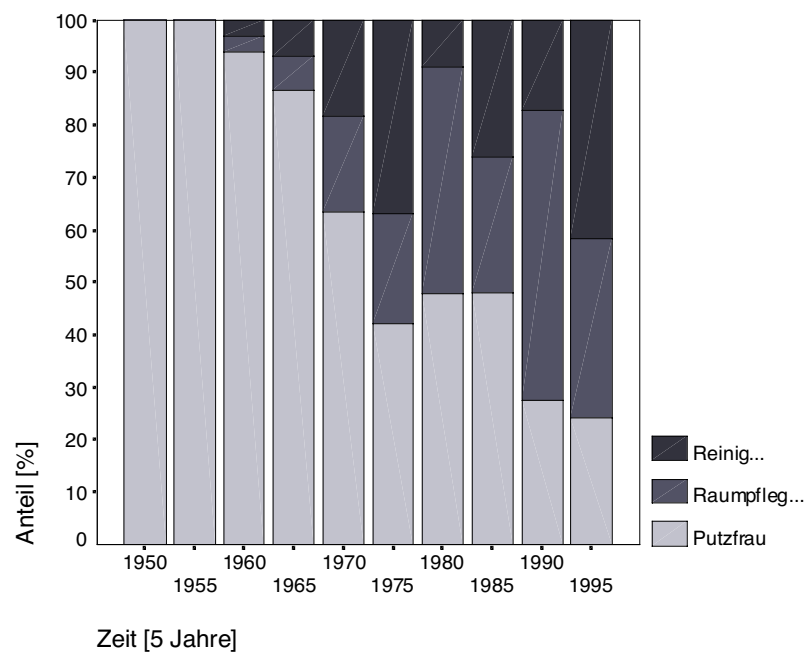

Relative Häufigkeit der Bezeichnungen "Putzfrau", "RaumpflegerIn" und solchen, die "Reinig..." enthalten (z.B. Reinigungsmitarbeiter, Reinigerin, Reinigungskraft, Frau für Ladenreinigung), pro fünf Jahre, in Stellenanzeigen 1950-1999. Insgesamt sind es 234 Fälle, davon 127 Nennungen von "Putzfrau".

Hier ist deutlich zu erkennen, dass die herkömmliche Berufsbezeichnung "Putzfrau" durch neue, "prestigeträchtigere" Bezeichnungen abgelöst wird.

Grafik 4: Zusammensetzung der erschlossenen Berufe, die mit der Bezeichnung "Mitarbeiter" resp. "Mitarbeiterin(nen)" aufgewertet werden

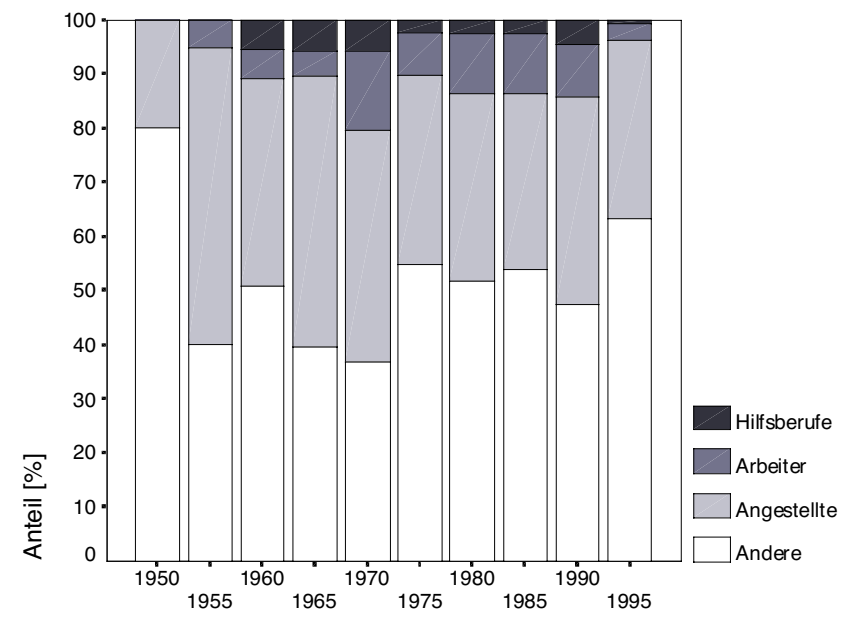

Zeit [5 Jahre]

Anteile der "Angestellten" (z.B. Bankangestellter, kaufmännische Angestellte, Kanzleiangestellter), "Arbeiter" (z.B. Fabrikarbeiterin, Lagerarbeiter, Metallarbeiter), "Hilfsberufe" (z.B. Magazingehilfe, Bürogehilfin, Hilfsmonteur, Packerhelfer) und "Andere", die unter der Bezeichnung "Mitarbeiter" resp. "Mitarbeiterin(nen)" in Stellenanzeigen 1950-1999 gesucht wurden. 